This item was submitted to Loughborough's Research Repository by the author.

Items in Figshare are protected by copyright, with all rights reserved, unless otherwise indicated.

\title{
Giant thermopower and figure of merit of semiconducting polaronic nanolayers
}

PLEASE CITE THE PUBLISHED VERSION

http://link.aps.org/doi/10.1103/PhysRevB.81.153204

PUBLISHER

(C) American Physical Society

VERSION

VoR (Version of Record)

LICENCE

CC BY-NC-ND 4.0

REPOSITORY RECORD

Alexandrov, A.S., and A.M. Bratkovsky. 2019. "Giant Thermopower and Figure of Merit of Semiconducting Polaronic Nanolayers”. figshare. https://hdl.handle.net/2134/6588. 
This item was submitted to Loughborough's Institutional Repository (https://dspace.lboro.ac.uk/) by the author and is made available under the following Creative Commons Licence conditions.

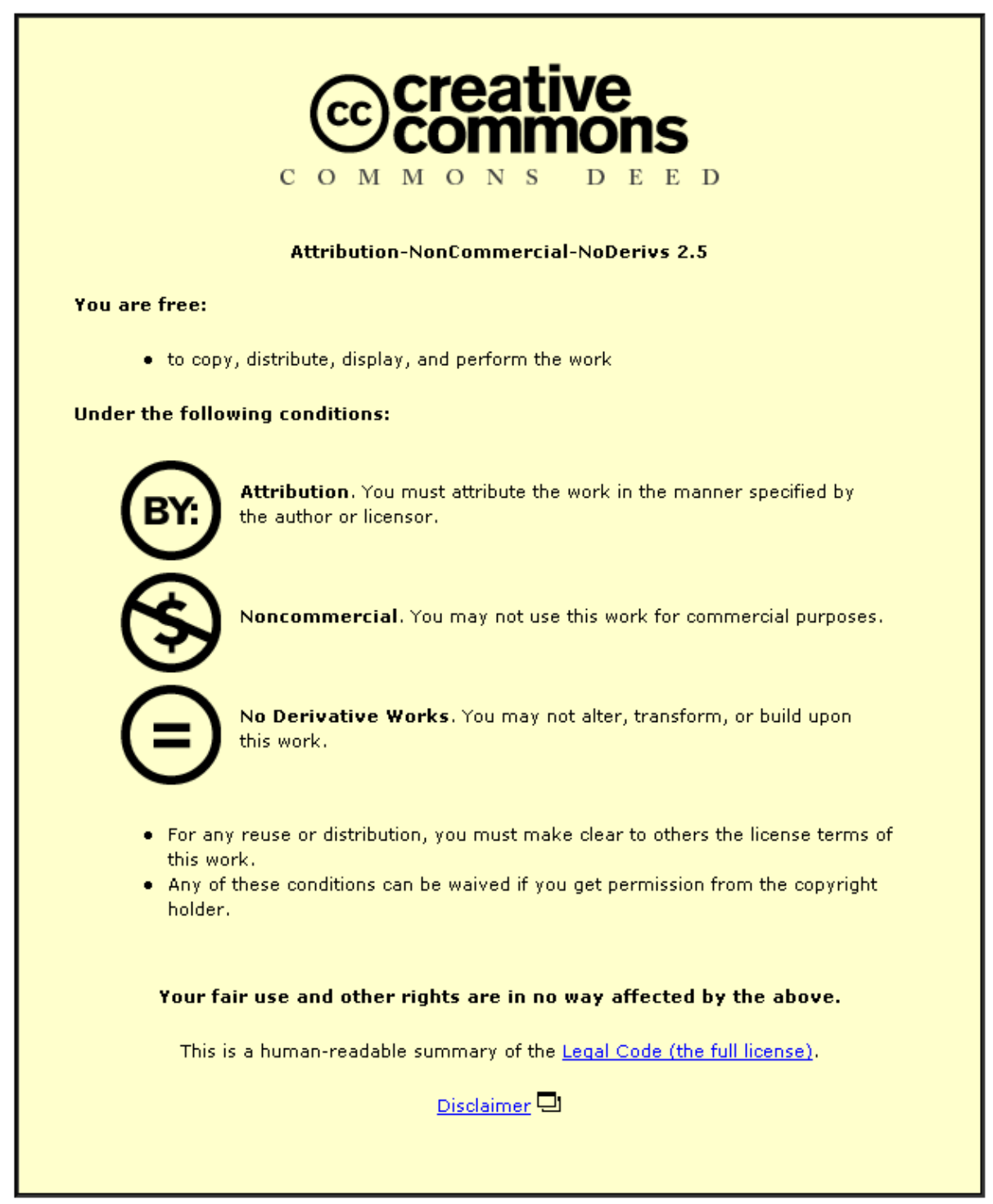

For the full text of this licence, please go to: http://creativecommons.org/licenses/by-nc-nd/2.5/ 


\title{
Giant thermopower and figure of merit of semiconducting polaronic nanolayers
}

\author{
A. S. Alexandrov ${ }^{1}$ and A. M. Bratkovsky ${ }^{2}$ \\ ${ }^{1}$ Department of Physics, Loughborough University, Loughborough LE11 3TU, United Kingdom \\ ${ }^{2}$ Hewlett-Packard Laboratories, 1501 Page Mill Road, Palo Alto, California 94304, USA
}

(Received 5 March 2010; published 29 April 2010)

\begin{abstract}
Polarons - electrons coupled with lattice vibrations - play a key role in transport and optical properties of many semiconductors. Here, we calculate the energy spectrum and thermopower of Fröhlich polarons confined to a potential well as a function of the well thickness. We show that the polaron mass enhancement in $2+\epsilon$ dimensions explains a giant thermoelectric power recently observed in doped semiconducting nanolayers (multiple quantum wells) and propose a route for enhancing the performance of thermoelectric energy nanoconverters by increasing their figure of merit by more than one order of magnitude.
\end{abstract}

DOI: 10.1103/PhysRevB.81.153204

PACS number(s): 71.38.-k, 72.10.Di, 72.20.Pa, 85.80.Fi

While the form of the electron-phonon (Fröhlich) Hamiltonian $^{1,2}$ is the same in all dimensions, the polaron mass, binding energy, and different response functions depend on the dimensionality for large polarons ${ }^{3-6}$ and on the dimensionality and crystal lattice structure for small polarons, ${ }^{7}$ especially in the intermediate coupling regime. In particular, directly following, ${ }^{8}$ the variational large polaron energy was calculated in Ref. 4 in different dimensions providing scaling relations valid to second order in the Fröhlich coupling constant $\alpha$. In the important case of a twodimensional (2D) confinement, the scaling relations take the form $^{2-4}$

$$
\begin{aligned}
E_{2 \mathrm{D}, p}(\alpha) & =\frac{2}{3} E_{3 \mathrm{D}, p}\left(\frac{3 \pi}{4} \alpha\right), \\
\frac{m_{2 \mathrm{D}}^{*}(\alpha)}{m_{2 \mathrm{D}}} & =\frac{m_{3 \mathrm{D}}^{*}\left(\frac{3 \pi}{4} \alpha\right)}{m_{3 \mathrm{D}}}, \\
\mu_{2 \mathrm{D}}(\alpha) & =\mu_{3 \mathrm{D}}\left(\frac{3 \pi}{4} \alpha\right)
\end{aligned}
$$

for the ground-state binding energy (polaron level shift, $E_{p}$ ), polaron mass, $m^{*}$, and the mobility, $\mu$ (if the scattering is dominated by optical phonons). Here $m$ is the band mass in a rigid lattice.

These scaling relations are instrumental for a qualitative understanding of physical properties of semiconducting layered nanostructures with polaronic carriers, which have a potential to provide a new route for realizing practical thermoelectric devices with the high figure of merit, $Z=S^{2} \sigma / \kappa$ $(S, \sigma$, and $\kappa$ are the thermoelectric Seebeck coefficient, and the electrical and thermal conductivities, respectively). Following a theoretical prediction by Hicks and Dresselhaus ${ }^{9}$ that layering can increase the figure of merit due to an increase in the density of states of confined carriers, different multiple quantum wells (MQWs) have been manufactured with an enhanced $Z$. More recently, Ohta et al. ${ }^{10}$ observed a giant enhancement of $S$ in $\mathrm{Nb}: \mathrm{SrTiO}_{3} / \mathrm{SrTiO}_{3}$ superlattices consisting of conducting $\mathrm{Nb}$-doped $\mathrm{SrTiO}_{3}$ (STO) nanolayers confined between insulating STO layers. With decreasing thickness of the conducting layer, the magnitude of $S$ increases to a value about five times that of the bulk Nb:STO, reaching almost $500 \mu \mathrm{V} / \mathrm{K}$ for the one unit-cell layer, which is much higher than what was anticipated theoretically. ${ }^{9}$

Doped STO has been recognized as a generic polaronic perovskite a long time ago. ${ }^{11}$ Some earlier and recent experimental observations of the optical conductivity in the bulk Nb-doped STO (Ref. 12) clearly reveal the evidence for the mid-infrared optical conductivity band provided by the polaron mechanism as in many other oxides. The effective mass of the charge carriers is obtained by analyzing the Drude spectral weight. Defining the mass renormalization of the charge carriers as the ratio of the total electronic spectral weight and the Drude spectral weight, a twofold mass enhancement is obtained, which is attributed to the electronphonon interaction (EPI). The missing spectral weight is recovered according to the sum rule ${ }^{13}$ in the mid-infrared optical conductivity band. This band results from EPI, traditionally associated with the polaronic nature of the charge carriers. The effective mass obtained from the optical spectral weights yields an intermediate electron-phonon coupling strength, $\alpha \simeq 3 .{ }^{12}$ Importantly, very recently Choi et al. ${ }^{14}$ observed a threefold mass enhancement in $\mathrm{Nb}: \mathrm{SrTiO}_{3} / \mathrm{SrTiO}_{3}$ superlattices compared with the bulk $\mathrm{Nb}$-doped STO as the $\mathrm{Nb}: \mathrm{SrTiO}_{3}$ layer thickness decreased from eleven to one unit cell. The authors of Ref. 14 have suggested that such an increase should be attributed to a dimensional crossover of a polaron from three-dimensional (3D) to 2D and seems to be associated with the strongly enhanced Seebeck coefficient. Here we propose a theory of the dimensional crossover of the large polaron from $3 \mathrm{D}$ to $2 \mathrm{D}$ providing detailed explanation of the giant thermopower enhancement in polaronic MQWs and predicting further possibilities for enhancing the performance of thermoelectric MQW devices.

We consider a polaron confined to an infinite potential well of the width $d$ in $z$ direction, such as $\mathrm{Nb}: \mathrm{SrTiO}_{3}$ layer merged into a bulk undoped $\mathrm{SrTiO}_{3}$. For large (or continuous, in other words) polarons the lattice structure is irrelevant $^{2}$ so that we adopt an effective band mass approximation and the canonical Fröhlich interaction ${ }^{1}$ of doped carriers with optical phonons in ionic insulators and also include the confinement potential $U(z)$, 


$$
\begin{aligned}
H= & -\frac{\hbar^{2} \nabla^{2}}{2 m}+\sum_{\mathbf{q}}\left(V_{\mathbf{q}} d_{\mathbf{q}} e^{i \mathbf{q} \cdot \mathbf{r}}+\text { H.c. }\right) \\
& +\hbar \omega \sum_{\mathbf{q}}\left(d_{\mathbf{q}}^{\dagger} d_{\mathbf{q}}+1 / 2\right)+U(z) .
\end{aligned}
$$

Here $d_{\mathbf{q}}\left(d_{\mathbf{q}}^{\dagger}\right)$ annihilates (creates) optical phonons with the frequency $\omega$. While EPI is moderate, one can apply the perturbation theory with respect to $V_{\mathbf{q}}$, which works well even in the intermediate coupling regime $\alpha \lesssim 2$ as verified by the Feynman all-coupling path-integral theory. ${ }^{2}$ The quantum states of a noninteracting electron and phonons are classified with the in-plane $2 \mathrm{D}$ electron wave vector, $\mathbf{k}_{\|}$and a subband index $n$, and the $3 \mathrm{D}$ phonon wave vector $\mathbf{q}$, respectively. The unperturbed state is the vacuum $|0\rangle$ of phonons and the electron plane wave $\left|\mathbf{k}_{\|}, n, 0\right\rangle=\frac{1}{L} e^{i \mathbf{k}_{\|} \cdot \vec{\rho}}|n\rangle|0\rangle$, where $\vec{\rho}$ is the inplane electron coordinate and $|n\rangle$ depends on $z$. The bare energy spectrum of the electron in a layer of the area $L^{2}$ is $E_{n \mathbf{k}_{\|}}=\frac{\hbar^{2} k_{\|}^{2}}{2 m}+\epsilon_{n}$, and $n=1,2, \ldots$, corresponds to quantized subbands due to confinement in $z$ direction, perpendicular to the layer. EPI couples the state with the energy $E_{n \mathbf{k}_{\|}}$and states of a single phonon with the momentum $\mathbf{q}$ and the electron with the energy $E_{n^{\prime} \mathbf{k}_{\|}-q_{\|}}$. The corresponding matrix element is

$$
\left\langle\mathbf{k}_{\|}-q_{\|}, n^{\prime} ; 1_{\mathbf{q}}\left|V_{\mathbf{q}}\right| \mathbf{k}_{\|}, n ; 0\right\rangle=\frac{2 \hbar \omega(\pi \alpha)^{1 / 2}\left\langle n^{\prime}\left|e^{i q_{z} z}\right| n\right\rangle}{q \Omega^{1 / 2}(2 m \omega / \hbar)^{1 / 4}},
$$

where $\Omega$ is the volume of the whole sample including the doped layer and the host bulk crystal. The renormalized second-order energy $\widetilde{E}_{n \mathbf{k}_{\|}}$is found as

$$
\begin{aligned}
\widetilde{E}_{n \mathbf{k}_{\|}}= & E_{n}\left(\mathbf{k}_{\|}\right)-\frac{4 \pi \alpha \hbar^{2} \omega^{2}}{(2 \pi)^{3}(2 m \omega / \hbar)^{1 / 2}} \sum_{n^{\prime}} \int d^{3} q \\
& \times \frac{\left|\left\langle n^{\prime}\left|e^{i q_{z} z}\right| n\right\rangle\right|^{2}}{\left(q_{\|}^{2}+q_{z}^{2}\right)\left[\hbar^{2} k_{\|} q_{\|} \cos \phi / m+\hbar^{2} q_{\|}^{2} / 2 m+\epsilon_{n^{\prime}}-\epsilon_{n}+\hbar \omega\right]} .
\end{aligned}
$$

We consider temperatures $k_{B} T$ small compared with the characteristic phonon energy $\hbar \omega$ and low densities of carries. In this case there is no imaginary part of $\widetilde{E}_{n \mathbf{k}_{\|}}$for sufficiently low-energy subbands $n$, which means that the momentum is conserved. Expanding over $k_{\|}$and replacing the sum over $n^{\prime}$ by an integral, one obtains $\widetilde{E}_{\mathbf{k}}=-\alpha \hbar \omega+\hbar^{2} k^{2} / 2 m_{3 \mathrm{D}}$ in the $3 \mathrm{D}$ system, $d \rightarrow \infty$, with the familiar 3D polaron mass, ${ }^{1}$ $m_{3 \mathrm{D}}^{*}=m /(1-\alpha / 6)$. Keeping only terms with $n=n^{\prime}=1$ and the matrix element $\left\langle n\left|e^{i q_{z} z}\right| n\right\rangle=1$ in Eq. (6), we find $\widetilde{E}_{\mathbf{k}_{\|}}$ $=-\pi \alpha \hbar \omega / 2+\hbar^{2} k_{\|}^{2} / 2 m_{2 \mathrm{D}}$ with the polaron mass $m_{2 \mathrm{D}}^{*}=m /(1$ $-\alpha \pi / 8)$ for a 2D layer, $d \rightarrow 0$. Both the polaron binding energy and the mass satisfy the scaling relations Eq. (1).

In the crossover region, where neither $3 \mathrm{D}$ nor $2 \mathrm{D}$ limits are applied, the spectrum is quantized into subbands,

$$
\tilde{E}_{n \mathbf{k}_{\|}}=\frac{\hbar^{2} k_{\|}^{2}}{2 m_{n}^{*}}+\epsilon_{n}-E_{p n},
$$

with the subband polaron energy shift $-E_{p n}$ and the subband in-plane polaron mass, $m_{n}^{*}$, found as

$$
\frac{E_{p n}}{\alpha \hbar \omega}=\frac{t}{\pi} \sum_{n^{\prime}} \int_{0}^{\infty} d x \int_{-\infty}^{\infty} d y \frac{x\left|\left\langle n^{\prime}\left|e^{i y u}\right| n\right\rangle\right|^{2}}{\left(x^{2}+y^{2}\right)\left(x^{2}+n^{\prime 2}-n^{2}+t^{2}\right)}
$$

and

$$
\begin{aligned}
\frac{m}{m_{n}^{*}}= & 1-\frac{2 \alpha t^{3}}{\pi} \sum_{n^{\prime}} \int_{0}^{\infty} d x \int_{-\infty}^{\infty} d y \\
& \times \frac{x^{3}\left|\left\langle n^{\prime}\left|e^{i y u}\right| n\right\rangle\right|^{2}}{\left(x^{2}+y^{2}\right)\left(x^{2}+n^{\prime 2}-n^{2}+t^{2}\right)^{3}},
\end{aligned}
$$

respectively. Here, we use dimensionless phonon momenta $y=q_{z} d / \pi, x=q_{\|} d / \pi, z$ coordinate $u=\pi z / d$ of the polaron confined to the layer of the thickness $d$, and dimensionless thickness of the layer, $t=q_{p} d / \pi$, where $q_{p}=(2 m \omega / \hbar)^{1 / 2}$ is the large polaron radius. As in Refs. 3,5 , and 6 , we confine polarons to the infinite potential well within $0<z<d$ so that the bare eigenfunctions and bare eigenvalues are $|n\rangle=(2 / d)^{1 / 2} \sin (\pi n z / d)$ and $\epsilon_{n}=\hbar^{2} \pi^{2} n^{2} /\left(2 m d^{2}\right)$, respectively. Then, integrating first over the transverse momentum $y$ to avoid complicated oscillating functions in Eqs. (8) and (9) yields

$$
\frac{E_{p n}}{\alpha \hbar \omega}=\frac{t}{\pi^{2}} \sum_{n^{\prime}=1}^{\infty} \int_{0}^{\infty} d x \frac{I_{n^{\prime} n}(x)}{x^{2}+n^{\prime 2}-n^{2}+t^{2}}
$$

and

$$
\frac{m}{m_{n}^{*}}=1-\frac{2 \alpha t^{3}}{\pi^{2}} \sum_{n^{\prime}=1}^{\infty} \int_{0}^{\infty} d x \frac{x^{2} I_{n^{\prime} n}(x)}{\left(x^{2}+n^{\prime 2}-n^{2}+t^{2}\right)^{3}}
$$

with

$$
I_{n n}(x)=\frac{2\left(\pi x+e^{-\pi x}-1\right)}{x^{2}}+\frac{\pi x}{x^{2}+4 n^{2}}+\frac{2\left(8 n^{2}+x^{2}\right)\left(1-e^{-\pi x}\right)}{\left(x^{2}+4 n^{2}\right)^{2}}
$$

for the diagonal contribution and

$$
I_{n^{\prime} n}(x)=\frac{\pi x}{x^{2}+k^{2}}+\frac{\pi x}{x^{2}+m^{2}}-\frac{2 x^{2}\left(m^{2}-k^{2}\right)^{2}\left[1-(-1)^{m} e^{-\pi x}\right]}{\left(x^{2}+m^{2}\right)^{2}\left(x^{2}+k^{2}\right)^{2}}
$$

for the off-diagonal terms, $n^{\prime} \neq n$, where $m=n^{\prime}+n$ and $k$ $=n^{\prime}-n\left(n^{\prime}, n=1,2, \ldots, \infty\right)$. Two last terms in the diagonal contribution, Eq. (12), and the last term in the off-diagonal contribution, Eq. (13) are numerically small, about a few percent of the remaining terms. Integration over $x$ and summation over $n^{\prime}$ in Eqs. (10) and (11) can be performed using any standard software. The polaron level shift and the mass for the lowest subband $(n=1)$ interpolate well between $2 \mathrm{D}$ and 3D limits, Figs. 1 and 2, for $t \rightarrow 0$ and $t \rightarrow \infty$, respectively, in agreement with Ref. 6 but in contrast to Ref. 5, where both limits are not obeyed. Both the shift and the mass are different for different subbands in $2+\epsilon$ crossover region as shown in Fig. 1. As can be seen in Fig. 1, one encounters the familiar flattening of the polaron dispersion and the breakdown of the perturbation theory at energies about the phonon energy ${ }^{15}$ for high-energy subbands. Importantly, the 

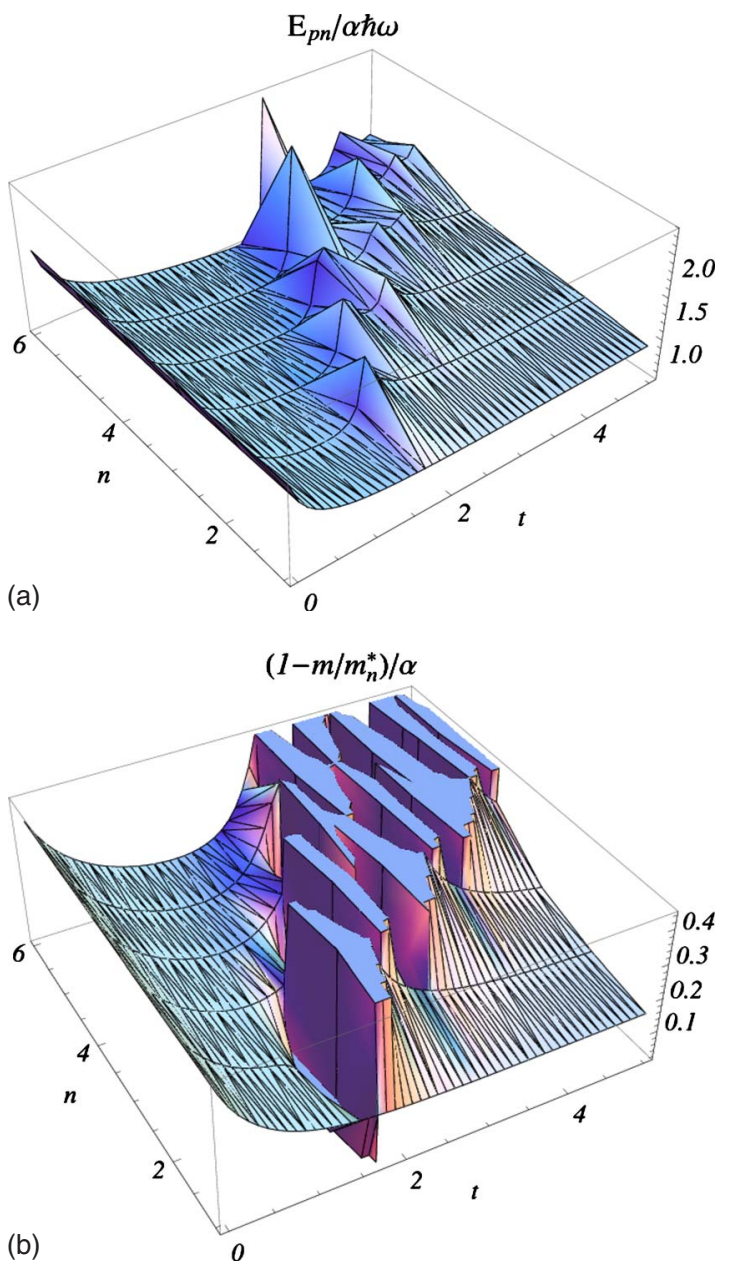

FIG. 1. (Color online) (a) Normalized polaron level shift $E_{p n} / \alpha \hbar \omega$ and (b) the relative polaron mass renormalization $\left(1-m / m_{n}^{*}\right) / \alpha$ for a number of subbands $n$ and different nanolayer thickness $t$ in the crossover region. A sudden increase in the shift and in the mass for sufficiently thick nanolayers $(t>2)$ and highenergy subbands is due to a breakdown of the perturbation expansion. polaron mass changes from its $2 \mathrm{D}$ value to the $3 \mathrm{D}$ value for $t$ in the range of about unity, Fig. 1. The experimental values of the effective thickness $t$ are about $t=0.16 N$, where $N$ is the number of atomic layers in the well, ${ }^{10,14}$ if we take $\hbar \omega$ $=0.05 \mathrm{eV}, m=m_{e}$, and the lattice constant $a=0.4 \mathrm{~nm}$, which are typical of STO. Hence, the experimental conditions are just right for a significant change in the polaron mass with the number of layers in $\mathrm{Nb}: \mathrm{SrTiO}_{3} / \mathrm{SrTiO}_{3}$ superlattices where $N$ changes from 1 to $11 .^{10,14}$ The threefold mass enhancement with decreasing thickness ${ }^{14}$ corresponds to $\alpha \approx 2.8$, Fig. 2 , which is close to optically measured value in the bulk Nb:SrTiO. ${ }^{12}$

Equations (10) and (11) and the Boltzmann theory allow us to analyze the dependence of different kinetic and thermodynamic properties on the confinement. In particular, applying the energy-independent relaxation-time $(\tau)$ approximation, one obtains the Seebeck coefficient as

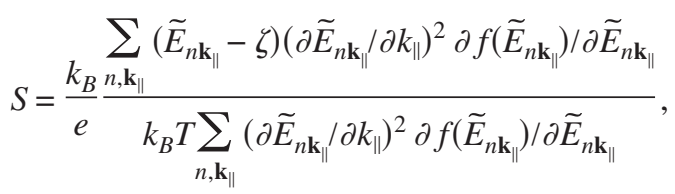

where $f(E)=1 /\left[1+\exp (E-\zeta) / k_{B} T\right]$ is the Fermi-Dirac distribution function. In the bulk system with $m_{3 \mathrm{D}}^{*}=2 m_{e}$, the Fermi energy is about $\zeta / k_{B}=1250 \mathrm{~K}$ for the polaron density $n_{p}=10^{21} \mathrm{~cm}^{-3}$ so that carriers are almost degenerate at room temperature. However, the system evolves from a degenerate to a classical polaron gas with decreasing nanolayer thickness because of the mass enhancement in the crossover region, Fig. 1. Hence one has to use the exact Fermi-Dirac statistics in this region. Integrating over momentum in Eq. (14) yields
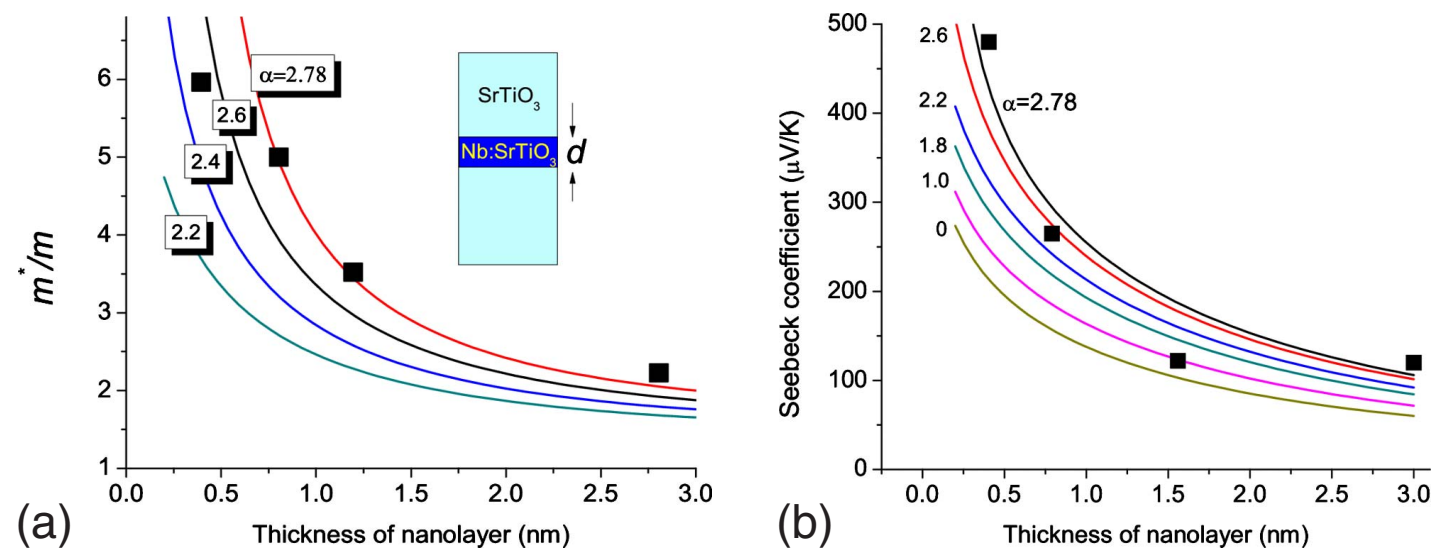

FIG. 2. (Color online) (a) Polaron mass $\mathrm{m}^{*} / \mathrm{m}$ of the lowest subband and (b) Seebeck coefficient $S$ in the crossover region for a few coupling constants $\alpha$ and $\hbar \omega=0.05 \mathrm{meV}, T=300 \mathrm{~K}, m=m_{e}$, and $n_{p}=1.66 \times 10^{20} \mathrm{~cm}^{-3}$ (solid lines) as the functions of the nanolayer thickness $d$ [inset in (a)]. Symbols correspond to the experimental mass measured optically in Ref. 14 and to the experimental Seebeck coefficient measured in Ref. 10. 


$$
\frac{e S}{k_{B}}=\frac{\sum_{n}\left[\frac{\pi^{2}}{3}+\left(\ln y_{n}\right)^{2}+2 L i_{2}\left(-\frac{1}{y_{n}}\right)-\ln \left(y_{n}\right) \ln \left(1+y_{n}\right)\right]}{\sum_{n} \ln \left(1+y_{n}\right)},
$$

where $y_{n}=\exp \left[\left(\zeta-\frac{\pi^{2} \hbar^{2} n^{2}}{2 m d^{2}}+E_{p n}\right) / k_{B} T\right]$ satisfies the following sum rule

$$
\sum_{n=1}^{\infty} m_{n}^{*} \ln \left(1+y_{n}\right)=\frac{\pi \hbar^{2} n_{p} d}{k_{B} T}
$$

and $L i_{2}(z)=\sum_{k=1}^{\infty} z^{k} / k^{2}$ is the dilogarithm function. Since $\hbar \omega / k_{B} T>1$ at room temperature for typical optical phonons in oxide ionic lattices, one can safely neglect all higher subband contributions in Eqs. (15) and (16) compared with the lowest subband term $n=1$ in the crossover region, $t<1$, avoiding the breakdown of the perturbation expansion, Fig. 1. The thermopower, calculated using this single-subband approximation, accounts rather well for the experiment ${ }^{10}$ with the same values of the EPI coupling, which accounts for the experimental polaron mass in Fig. 2. The carrier density turns out significantly lower compared with the chemical density presumably due to a partial localization of polarons by impurities. Possible interface effects on phonons ${ }^{6}$ may further enhance the thermopower.

Finally, let us discuss the confinement enhancement of the figure of merit. In semiconducting MQWs such as $\mathrm{Nb}: \mathrm{SrTiO}_{3} / \mathrm{SrTiO}_{3}$ superlattices, the thermal conductivity is mainly due to phonons and largely independent on doping and confinement. If we take $S \propto m^{*}$ then, using the scaling Eq. (1), the maximum confinement enhancement of $Z$ is estimated as $Z_{2 \mathrm{D}} / Z_{3 \mathrm{D}}$ $=m_{3 \mathrm{D}}^{*}(3 \pi \alpha / 4)^{2} \mu_{3 \mathrm{D}}(3 \pi \alpha / 4) / m_{3 \mathrm{D}}^{*}(\alpha)^{2} \mu_{3 \mathrm{D}}(\alpha)$, if polarons are scattered by absorption of optical phonons. In this case, the mobility depends on the coupling strength as ${ }^{16} \mu_{3 \mathrm{D}} \propto[1$ $\left.-\alpha / 6+\mathcal{O}\left(\alpha^{2}\right)\right] / \alpha$ so that the enhancement, $Z_{2 \mathrm{D}} / Z_{3 \mathrm{D}}$ $=(4 / 3 \pi)(1-\alpha / 6) /(1-\pi \alpha / 8)$, is only 1.3 for $\alpha=2$. How ever, at temperatures well below the characteristic optical phonon temperature $\hbar \omega / k_{B}$, the number of thermal phonons is exponentially small so that the polarons are mainly scattered off neutral impurities since the impurity potential is screened by heavy polarons at substantial doping. The scattering time off neutral impurities increases with the polaron mass as $\tau \propto\left(m^{*}\right)^{2}$ (Ref. 17) so that the mobility is proportional to the mass, $\mu \propto m^{*}$. In this case the confinement enhancement of the figure of merit may be giant. For the coupling $\alpha=2, Z_{2 \mathrm{D}} / Z_{3 \mathrm{D}}=(1-\alpha / 6)^{3} /(1-\pi \alpha / 8)^{3} \approx 30$ is much larger than in a free-electron layered system. ${ }^{9}$ This prediction provides further route for enhancing the performance of thermoelectric energy converters.

To conclude, we have developed the theory of polaron crossover from $3 \mathrm{D}$ to $2 \mathrm{D}$. In the crossover region the polaron energy spectrum is quantized into size subbands with the subband binding energy and polaron mass strongly dependent on the size of the confinement nanolayer, when the thickness of the layer is comparable or less than the large polaron radius, $d \leq 1 / q_{p}$. We have shown that this condition is satisfied in $\mathrm{Nb}: \mathrm{SrTiO}_{3} / \mathrm{SrTiO}_{3}$ superlattices and proposed a detailed explanation of the giant thermopower enhancement observed in these structures. ${ }^{10,14}$ The theory predicts a giant confinement enhancement of the figure of merit in those polaronic MQWs where the scattering of polarons is dominated by neutral impurities.

We greatly appreciate illuminating discussions with $\mathrm{J}$. T. Devreese, T. W. Noh, and J. Tempere, and the EPSRC (U.K.) support of this work (Grant No. EP/H004483).
${ }^{1}$ H. Fröhlich, Adv. Phys. 3, 325 (1954).

${ }^{2}$ A. S. Alexandrov and J. T. Devreese, Advances in Polaron Physics (Springer, New York, 2009).

${ }^{3}$ S. Das Sarma, Phys. Rev. B 27, 2590 (1983).

${ }^{4}$ F. M. Peeters and J. T. Devreese, Phys. Rev. B 36, 4442 (1987), and references therein.

${ }^{5}$ F. Comas, C. Trallero-Giner, and R. Riera, Phys. Rev. B 39, 5907 (1989).

${ }^{6}$ Guo-qiang Hai, F. M. Peeters, and J. T. Devreese, Phys. Rev. B 42, 11063 (1990), and references therein.

${ }^{7}$ J. P. Hague, P. E. Kornilovitch, A. S. Alexandrov, and J. H. Samson, Phys. Rev. B 73, 054303 (2006).

${ }^{8}$ R. P. Feynman, Phys. Rev. 97, 660 (1955).

${ }^{9}$ L. D. Hicks and M. S. Dresselhaus, Phys. Rev. B 47, 12727 (1993).

${ }^{10}$ H. Ohta, S. Kim, Y. Mune, T. Mizoguchi, K. Nomura, S. Ohta, T. Nomura, Y. Nakanishi, Y. Ikuhara, M. Hirano, H. Hosono, and
K. Koumoto, Nature Mater. 6, 129 (2007).

${ }^{11}$ D. M. Eagles and P. Lalousis, J. Phys. C 17, 655 (1984), and references therein.

${ }^{12}$ J. L. M. van Mechelen, D. van der Marel, C. Grimaldi, A. B. Kuzmenko, N. P. Armitage, N. Reyren, H. Hagemann, and I. I. Mazin, Phys. Rev. Lett. 100, 226403 (2008), and references therein.

${ }^{13}$ J. T. Devreese, L. Lemmens, and J. Van Royen, Phys. Rev. B 15, 1212 (1977).

${ }^{14}$ W. Choi, H. Ohta, S. Moon, Y. Lee, and T. Noh, arXiv:0906.5391 (unpublished).

${ }^{15}$ Y. B. Levinson and E. I. Rashba, Rep. Prog. Phys. 36, 1499 (1973).

${ }^{16}$ D. C. Langreth and L. P. Kadanoff, Phys. Rev. 133, A1070 (1964).

${ }^{17}$ A. Anselm, Introduction to Semiconductor Theory (PrencticeHall, Englewood Cliffs, NJ, 1981). 